\title{
Effect of Micro-Dimples on Hydrodynamic Lubrication of Textured Sinusoidal Roughness Surfaces
}

Jing-Hu Ji', Cai-Wei Guan and Yong-Hong Fu

\begin{abstract}
Surface texturing has been applied to improving the tribological performance of mechanical components for many years. Currently, the researches simulate the film pressure distribution of textured rough surfaces on the basis of the average flow model, and however the influence of roughness on the film pressure distribution could not be precisely expressed. Therefore, in order to study the hydrodynamic lubrication of the rough textured surfaces, sinusoidal waves are employed to characterize untextured surfaces. A deterministic model for hydrodynamic lubrication of microdimple textured rough surfaces is developed to predict the distribution of hydrodynamic pressure. By supplementing with the JFO cavitation boundary, the load carrying capacity of the film produced by micro-dimples and roughness is obtained. And the geometric parameters of textured rough surface are optimized to obtain the maximum hydrodynamic lubrication by specifying an optimization goal of the load carrying capacity. The effect of roughness on the hydrodynamic pressure of surface texture is significant and the load carrying capacity decreases with the increase of the roughness ratio because the roughness greatly suppresses the hydrodynamic effect of dimples. It shows that the roughness ratio of surface may be as small as possible to suppress the effect of hydrodynamic lubrication. Additionally, there are the optimum values of the micro-dimple depth and area density to maximize the load carrying capacity for any given value of the roughness ratio. The proposed approach is capable of accurately reflects the influence of roughness on the hydrodynamic pressure, and developed a deterministic model to investigate the hydrodynamic lubrication of textured surfaces.
\end{abstract}

Keywords: Hydrodynamic lubrication, Surface texturing, Micro-dimple, Roughness surface, Sinusoidal wave

\section{Introduction}

Surface texturing has become an efficient process of generating various texture patterns to improve tribological properties of mechanical components in the last decades. In the development of surface texturing technology there have been great efforts to reveal the role of geometrical parameters of surface texture in tribology [1]. Specially, the research on hydrodynamic lubrication of surface texture has attracted much attention and long been a great challenge to scholars and engineers [2].

The development of mechanical engineering has created a greater demand for understanding and utilizing

\footnotetext{
*Correspondence: andyjee@163.com

School of Mechanical Engineering, Jiangsu University, Zhenjiang 212013, China
}

the lubrication and anti-friction mechanisms of surface micro-geometry [3, 4]. Optimization design of surface textures is one of the key factors for improving the tribological performance of mechanical components [5]. Compared with the experimental investigation, the lubrication performance of textures can be precisely quantified by numerical analysis [6].

Based on the operating conditions and the contact geometry of mechanical components, the proper analytical model can be established to simulate the distribution of lubricant film thickness and pressure of the textured surfaces $[7,8]$, with the geometrical parameters of surface textures analyzed systematically by the numerical simulation. For example, a simplified model of "piston/cylinder" with micro-dimples was developed to predict the tribological performance of reciprocating 
automotive components by Ronen et al. [9]. The threedimensional instantaneous pressure distribution over the textured surface was simulated to analyze the effect of micro-dimples on the hydrodynamic lubrication. It is shown that the geometric parameters of micro-dimples, such as area density and dimple depth, have a critical influence on the distribution of film pressure, especially of the dimple depth.

Furthermore, the effect of different shapes of textures on the hydrodynamic pressure could also be analyzed by the numerical studies $[10,11]$. Therefore, other than an analysis of a single type of texture [12], the numerical simulation may be a good way to evaluate the pros and cons of various types of textures under some typical operating conditions [13]. Besides, the effect of textured area on the hydrodynamic lubrication could also be analyzed by numerical analysis $[14,15]$. The physical mechanisms of two types of surface texturing concepts to generate hydrodynamic pressure, full periodic texturing and partial texturing, could be revealed by numerical analysis. There are the micro-dimple "individual effect" and "collective effect" corresponding to the full periodic texturing and partial texturing, respectively.

Based on the above review, the key geometrical parameters exerting considerable effects on the lubrication could be clarified by the distribution of film pressure [16]. Consequently, a series of studies have been done to investigate the hydrodynamic lubrication of surface textures [17-20]. However, in order to make it easier for the analytical model to be developed and solved, the influences of surface roughness have almost been ignored. And yet, the effect of surface roughness on hydrodynamic lubrication should not be ignored as the surface roughness and the average thickness of lubrication film are in the same magnitude [21, 22].

The average flow model has been introduced to study the influence of surface roughness on the hydrodynamic lubrication of textured surfaces by Qiu et al. [23]. However, the average flow model was proposed by means of statistical theory, and the film pressure distribution could not reflect precisely the micro-surface structure's influence on the film pressure [24]. Thus, those kinds of studies have always been controversial in the tribological community [25]. By computing the film pressure through rough surfaces which were simulated with sinusoidal waves, the tribological mechanisms of the surfaces could be known more.

In this paper, the hydrodynamic lubrication of the relative movement of two parallel sliders are investigated with provision for surface roughness. Sinusoidal waves $[21,24]$ are employed to characterize the micro-structure of rough surfaces, with an analytical model of textured rough surfaces with micro-dimples developed to predict the hydrodynamic pressure.

\section{Analytical Model}

The model of two parallel sliders is presented in Figure 1. Supposing the above slider is stationary and has regular rough surface, and the underneath slider is textured with micro-dimples and moving at a steady speed of $U$ relative to the above slider.

Thousands of micro-dimples are evenly distributed in the textured surface, and each dimple is assumed by a spherical segment. The base radius and depth of the dimple are denoted by $r_{p}$ and $h_{p}$, respectively. Each dimple is supposed to locate in the center of a square cell of sides $2 r_{0} \times 2 r_{0}$, as shown in Figure 2 .

Based on the assumption that the parallel sliders are separated by a film of constant thickness $h_{0}$, that the lubricant is a Newtonian fluid with a constant viscosity $\eta$, and that the flow is considered laminar. Therefore, the generalized Reynolds equation for the hydrodynamic pressure can be given in the following form:

$$
\frac{\partial}{\partial x}\left(\rho h^{3} \frac{\partial p}{\partial x}\right)+\frac{\partial}{\partial y}\left(\rho h^{3} \frac{\partial p}{\partial y}\right)=6 \eta U \frac{\partial \rho h}{\partial x},
$$

where $h$ is the film thickness, $p$ is the film pressure, $U$ is the velocity, $x$ is the longitudinal direction Cartesian coordinate, $y$ is the lateral direction Cartesian coordinate, $\eta$ is the dynamic viscosity, $\rho$ is the density.

In the present paper, sinusoidal waves are employed to represent the rough surface, as expressed geometrically in the following form:

$$
R(x, y)=A_{0}-A_{0} \sin \left(\frac{2 \pi}{w_{x}}\right) \sin \left(\frac{2 \pi}{w_{y}}\right),
$$

where $A_{0}$ is the amplitude of the sinusoidal wave, $w_{x}$ is the wavelength in $x$ direction, $w_{y}$ is the wavelength in $y$ direction.

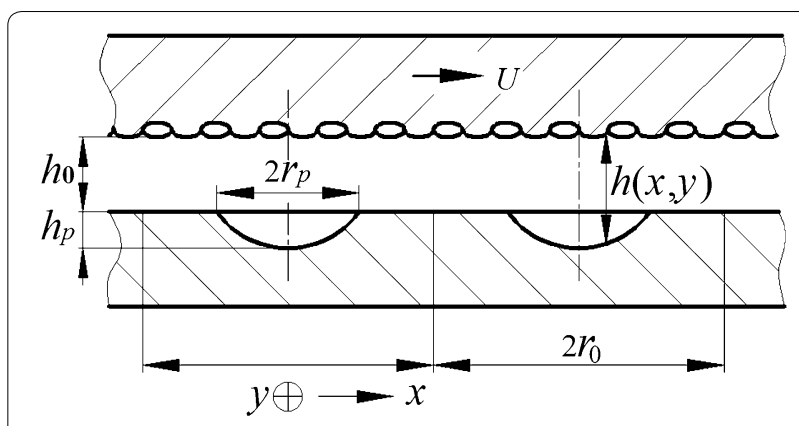

Figure 1 Geometrical model of two textured parallel sliders 


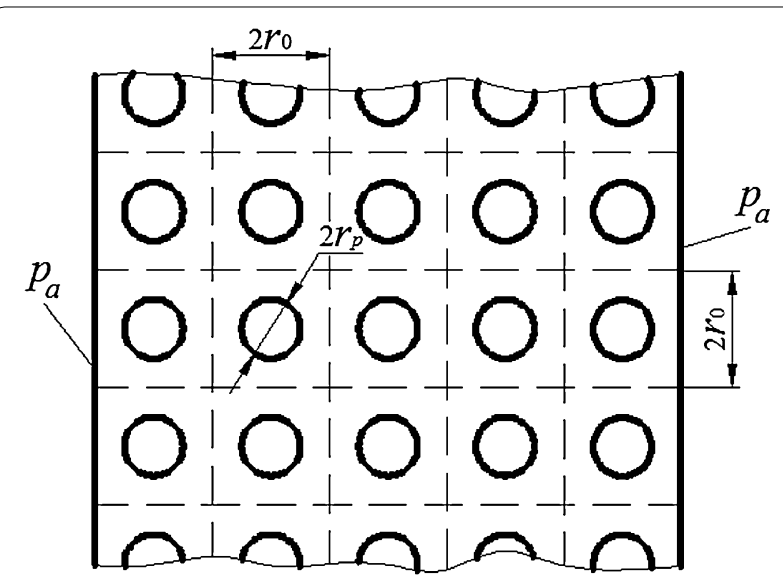

Figure 2 Geometrical distribution of the micro-dimples

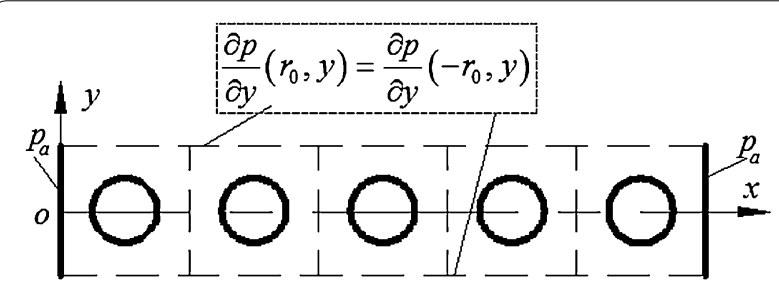

Figure 3 Dimples column and its spherical conditions

The dimple distribution in the surface of slider can be composed of thousands of column lists with a width of $2 r_{0}$ in $y$ direction, as shown in Figure 2. To simplify the operation, the slider is considered to be infinitely wide and the sinusoidal wavy has a periodical change in the surface perpendicular to the sliding direction. Based on these assumptions, the impact of the end effects in this direction can't be considered in the process of numerical simulation, and the pressure distribution is spatially modulated with a cycle equal to $2 r_{0}$. Therefore, as can be seen from Figure 3, it is enough to consider a dimple column along $x$ direction.

Based on the assumption of spherical dimples, the geometrical morphology of the textured smooth surface in the dimple column is given by: where $k$ is the number of dimple's rank, $k=1,2,3, \ldots, N_{p}$, $N_{p}$ is the number of dimples in $x$ direction.

Therefore, the film thickness at any point of the dimples column between the textured smooth and rough surfaces can be obtained from the simultaneous Eqs. (2) and (3), as shown in the following form:

$$
h(x, y)=h_{0}+R(x, y)+s(x, y),
$$

where $h_{0}$ denotes the minimum film thickness between the textured smooth and rough surfaces.

As shown in Figure 3, the slider boundaries pressure are equal to the atmosphere pressure, $p_{\mathrm{a}}$, in $x$ direction. Besides, the periodical pressure is applied in the textured rough surface. So the pressure boundary conditions can be given in the following equations:

$$
\left\{\begin{array}{c}
p(0, y)=p\left(l_{x}, y\right)=p_{a}, \\
\frac{\partial p}{\partial y}\left(x,-r_{0}\right)=\frac{\partial p}{\partial y}\left(x, r_{0}\right), \\
p\left(x,-r_{0}\right)=p\left(x, r_{0}\right)
\end{array}\right.
$$

Based on the main fundamental principle of the Jakobsson-Floberg-Olsson (JFO) cavitation theory [26], the fractional-film content $\left(\theta=\rho_{c} / \rho\right)$ is applied to the Reynolds equation, and a "universal" partial differential equation (PDE) covering both the full-film and cavitation regions can be proposed for simplification, generalization and improvement in dealing with the cavitation algorithm. However, in order to make the resulting PDE consistent with the uniform pressure assumption within the cavitation region, a switch $g$ is introduced by Elrod [26], as shown in the following function:

$$
g=\left\{\begin{array}{l}
0, \theta<1 \\
1, \theta \geq 1
\end{array}\right.
$$

And the resulting universal partial differential equation can be written as follows:

$$
\frac{\partial}{\partial x}\left(\beta h^{3} g \frac{\partial \theta}{\partial x}\right)+\frac{\partial}{\partial y}\left(\beta h^{3} g \frac{\partial \theta}{\partial y}\right)=6 \eta U \frac{\partial \theta h}{\partial x},
$$

$$
s(x, y)=\left\{\begin{array}{c}
\sqrt{\left(\frac{h_{p}^{2}+r_{p}^{2}}{2 h_{p}}\right)^{2}-\left\{\left[x-(2 k-1) r_{0}\right]^{2}+\left[y-r_{0}\right]^{2}\right\}-\frac{r_{p}^{2}-h_{p}^{2}}{2 h_{p}},} \\
{\left[x-(2 k-1) r_{0}\right]^{2}+\left[y-r_{0}\right]^{2} \leq r_{p}^{2},}
\end{array}\right.
$$


where $\beta$ is the bulk modulus of the lubricant, $\beta=\rho \frac{\partial p}{\partial \rho}$.

And the pressure can be calculated from the fractionalfilm content $\theta$ in the following function:

$$
p=\left\{\begin{array}{l}
p_{c}+\beta \ln \theta, \theta>1, \\
P_{c}, \theta<1
\end{array}\right.
$$

In order to facilitate the numerical simulation, Eq. (7) would be reduced to a dimensionless form, with the dimensionless variables defined as follows:

$$
X=\frac{x}{r_{c}}, Y=\frac{y}{r_{c}}, H=\frac{h}{h_{c}}, P=\frac{p}{p_{\mathrm{a}}},
$$

where $h_{c}$ is the virtual reference quantity of the dimple depth, $H$ is the dimensionless film thickness, $P$ is the dimensionless pressure, $r_{c}$ is the virtual reference quantity of the dimple radius, $X$ is the dimensionless longitudinal direction Cartesian coordinate, $Y$ is the dimensionless lateral direction Cartesian coordinate.

Substituting Eq. (10) into Eq. (7), the dimensionless Reynolds equation can be obtained in the following form:

$$
\frac{\partial}{\partial X}\left(g H^{3} \frac{\partial \theta}{\partial X}\right)+\frac{\partial}{\partial Y}\left(g H^{3} \frac{\partial \theta}{\partial Y}\right)=\Lambda \frac{\partial \theta H}{\partial X},
$$

where $\Lambda$ is the operating parameters, $\Lambda=\frac{6 \eta U r_{c}}{\beta h_{c}^{2}}$.

Subsequently, it is necessary to change Eq. (2) and Eq. (3) into the dimensionless forms. Then, the dimensionless sinusoidal wavy equation, $R^{\prime}$, and the geometrical morphology of the textured smooth surface equation, $S$, are given as follows:

$$
R^{\prime}(x, y)=\sigma-\sigma \sin \left(\frac{2 \pi}{W_{x}}\right) \sin \left(\frac{2 \pi}{W_{y}}\right),
$$

dimensionless side of imaginary rectangular cell, $R_{0}=\frac{r_{0}}{h_{c}}$, $\varepsilon$ is the ratio of $r_{c}$ over $h_{c}, \varepsilon=\frac{r_{c}}{h_{c}}$.

Hence, the dimensionless film thickness equation, $H(X, Y)$, is given by:

$$
H(X, Y)=H_{0}+R^{\prime}(X, Y)+S(X, Y),
$$

where $H_{0}$ is the dimensionless minimum film thickness, $H_{0}=\frac{h_{0}}{h_{c}}$.

And the dimensionless boundary conditions are given as follows:

$$
\left\{\begin{array}{c}
P(0, Y)=P\left(L_{x}, Y\right)=1, \\
\frac{\partial P}{\partial Y}\left(X,-R_{0}\right)=\frac{\partial P}{\partial Y}\left(X, R_{0}\right), \\
P\left(X,-R_{0}\right)=P\left(X, R_{0}\right),
\end{array}\right.
$$

where $L_{x}$ is the dimensionless slider length in $x$ direction, $L_{x}=\frac{l_{x}}{l_{c}}$

By using the multigrid method, the Reynolds equation, Eq. (10), is solved coupling with appropriate boundary conditions and the film thickness equations. For details, please refer to Venner et al. [27], Fesanghary et al. [28] and Ji et al. [29].

Once the fractional-film content $\theta$ is obtained by solving the analytical model, the dimensionless film pressure $P$ can be calculated from the following function:

$$
P=\left\{\begin{array}{l}
P_{c}+\bar{\beta} \ln \theta, \theta \geq 1, \\
P_{c}, \quad \theta<1,
\end{array}\right.
$$

where $P_{c}$ is the dimensionless cavitation pressure, $P_{c}=\frac{p_{c}}{p_{a}}, \bar{\beta}$ is the dimensionless bulk modulus of the lubricant, $\bar{\beta}=\frac{\beta}{p_{a}}$.

$$
\begin{aligned}
& S(X, Y) \\
& =\left\{\begin{array}{c}
\sqrt{\left(\frac{H_{p}^{2}+\varepsilon^{2} R_{p}^{2}}{2 H_{p}}\right)^{2}-\left\{\left[X-(2 k-1) R_{0}\right]^{2}+\left[Y-R_{0}\right]^{2}\right\}-\frac{\varepsilon^{2} R_{p}^{2}-H_{p}^{2}}{2 H_{p}}}, \\
{\left[X-(2 k-1) R_{0}\right]^{2}+\left[Y-R_{0}\right]^{2}<R_{p}^{2},} \\
0, \quad\left[X-(2 k-1) R_{0}\right]^{2}+\left[Y-R_{0}\right]^{2} \geq R_{p}^{2},
\end{array}\right.
\end{aligned}
$$

where $\sigma$ is the dimensionless amplitude of the sinusoidal wave, $\sigma=\frac{A_{0}}{h_{c}}, W_{x}$ is the dimensionless wavelengths of the sinusoidal wave in $x$ direction, $W_{x}=\frac{w_{x}}{r_{c}}, W_{y}$ is the dimensionless wavelengths of the sinusoidal wave in $y$ direction, $W_{y}=\frac{h_{p}}{h_{c}}, H_{y}$ is the dimensionless dimple depth, $H_{p}=\frac{h_{p}}{h_{c}}$, $R_{p}$ is the dimensionless dimple radius, $R_{p}=\frac{r_{p}}{l_{c}}, R_{0}$ is the
The dimensionless load carrying capacity, $W$, in the dimples column is shown in the following equation:

$$
W=\int_{-R_{0}}^{R_{0}} \int_{0}^{L_{x}} P \mathrm{~d} X \mathrm{~d} Y .
$$

And the equation for calculating the dimensionless average pressure, $P_{a v}$, can be obtained as follows: 


$$
P_{a v}=\frac{W}{2 R_{0} \cdot L_{x}} .
$$

\section{Results and Discussion}

Previous researches $[13,28]$ indicated that the dimensionless average pressure is a good standard of hydrodynamic lubrication assessment of surface texture. So a series of parametric analysis is made to investigate the influence of various parameters on the dimensionless average pressure, as shown in the following forms:

1. Number of dimples, $N_{p}$;

2. Roughness ratio, $\sigma\left(A_{0} / h_{\mathrm{c}}\right)$;

3. Dimensionless wavelengths, $W_{x}$ or $W_{y}$;

4. Dimple aspect ratio, $\delta\left(H_{\mathrm{p}} / H_{0}\right)$;

5. Area density of dimples, $S_{\mathrm{p}}$.

In order to investigate the influence of the geometrical parameters of micro-dimple and sinusoidal wavy rough surface on the hydrodynamic pressure, the pressure distribution will be computed according to the conditions in Table 1 in the following section, if not mentioned otherwise.

\subsection{Effect of Number of Dimples on Dimensionless Average Pressure}

Figure 4 shows the typical dimensionless film thickness and pressure distributions over the dimples column for $N_{p}=1$. It is clear that the pressures do not drop to zero along the $y$ direction because of the interaction between neighboring dimples. The hydrodynamic lubrication of micro-dimples was also studied by numerical analysis [10-12]. In these researches, the analytical models were developed to study the hydrodynamic lubrication of textured surfaces with a dimple only. It is obvious that

Table 1 Input parameters for case study

\begin{tabular}{ll}
\hline Parameters & Value \\
\hline Minimum film thickness $h_{0}(\mu \mathrm{m})$ & 2 \\
Reference value of film thickness $h_{c}(\mu \mathrm{m})$ & 1 \\
Reference value of dimple radius $r_{c}(\mu \mathrm{m})$ & 50 \\
Amplitude of the sinusoidal wave $A_{0}(\mu \mathrm{m})$ & 0.1 \\
Wavelengths of the sinusoidal wave $w_{x}$ or $w_{y}(\mu \mathrm{m})$ & 10 \\
Dimple radius $r_{p}(\mu \mathrm{m})$ & 50 \\
Dimple depth $h_{p}(\mu \mathrm{m})$ & 5 \\
Area density $S_{p}$ & 0.2 \\
Dynamic viscosity $\eta(\mathrm{mPa} \cdot s)$ & 3.5 \\
Sliding speed $U(\mathrm{~m} / \mathrm{s})$ & 10 \\
Ambient pressure $p_{a}(\mathrm{~Pa})$ & $1 \times 10^{5}$ \\
Cavitation pressure $p_{c}(\mathrm{~Pa})$ & $1 \times 10^{5}$ \\
Bulk modulus $\beta(\mathrm{Pa})$ & $1 \times 10^{8}$ \\
\hline
\end{tabular}

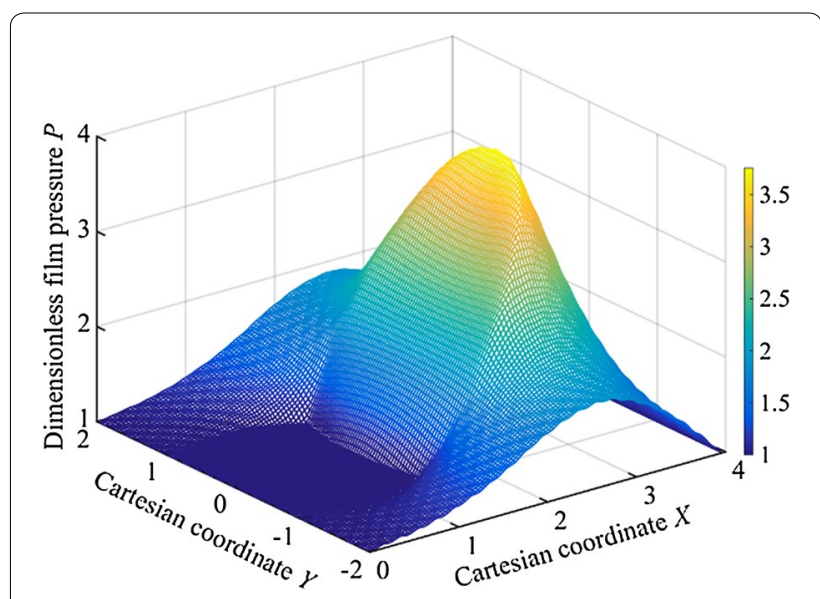

Figure 4 Hydrodynamic pressure over dimples column for $N_{p}=1$

the interaction between neighboring dimples in only one of the two directions had been considered in the previous attempts. Through a comparison and analysis of the film pressure distribution with the results obtained by Refs. [10-12], the interaction between neighboring dimples cannot be omitted in the process of numerical simulation. Figure 5 shows the pressure distribution over the dimples column for $N_{p}=7$. It is clearly visible that the pressure distribution of each dimple is strongly affected by its neighboring dimples. Comparing this with that in Figure 4, it is clear that the pressures do not drop to zero along the $x$ direction because of the interaction between neighboring dimples. Therefore, the interactions between neighboring dimples along the $x$ directions cannot be neglected in calculating the pressure distribution of surface texture, contrary to calculation in Refs. [10-12]. Besides, the pressure distribution is heavily influenced by the pressures at the end boundaries of the computational area in the $x$ direction. Thus, it can be concluded that the influence of boundaries pressure should not be neglected. The pressure distribution for 12 dimples is shown in Figure 6. It is observed that the influence of the pressure at boundaries becomes weak as the number of dimple increases in the computational area.

Figure 7 presents the effect of the number of dimples in the $x$ direction, with $N_{p}$ on the dimensionless average pressure, $P_{a v}$, for several values of the area density of the dimples. It can be seen clearly from the figure that $P_{a v}$ increases with the number of dimples increasing. However, the effect of $N_{\mathrm{p}}$ diminishes when the value of number of dimples increases. Furthermore, the rate of increase of $P_{a v}$ is very small when $N_{p} \geq 12$. Therefore, in order to reduce the computational time as well as to improve the efficiency and accuracy, the number of dimples is set as $N_{p}=12$. 


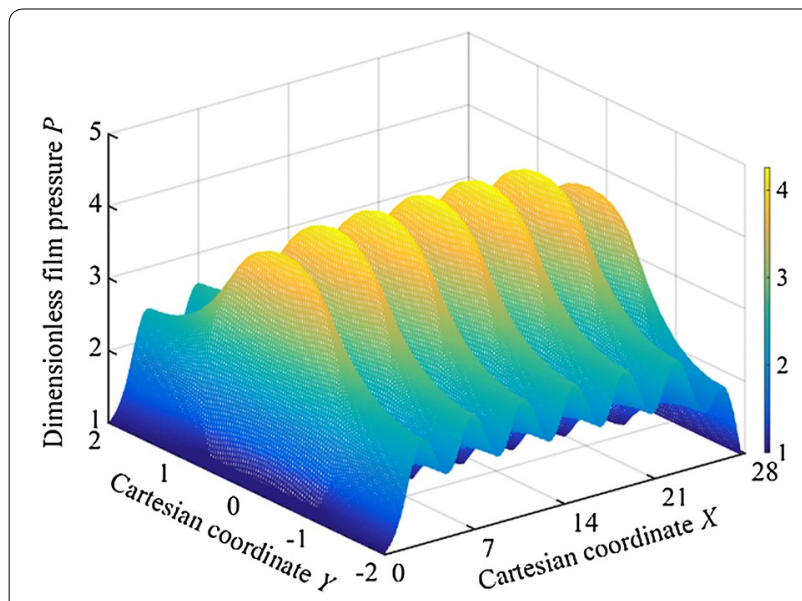

Figure 5 Hydrodynamic pressure over dimples column for $N_{p}=7$

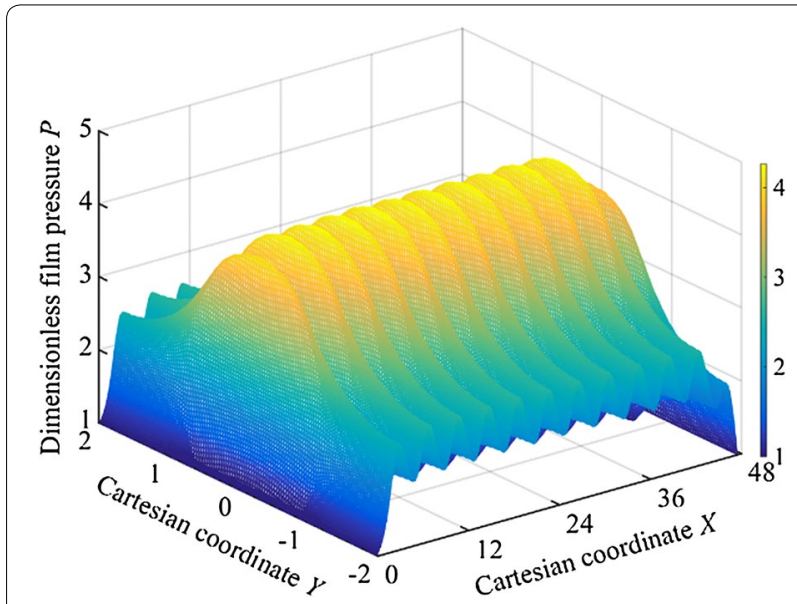

Figure 6 Hydrodynamic pressure over dimples column for $N_{p}=12$

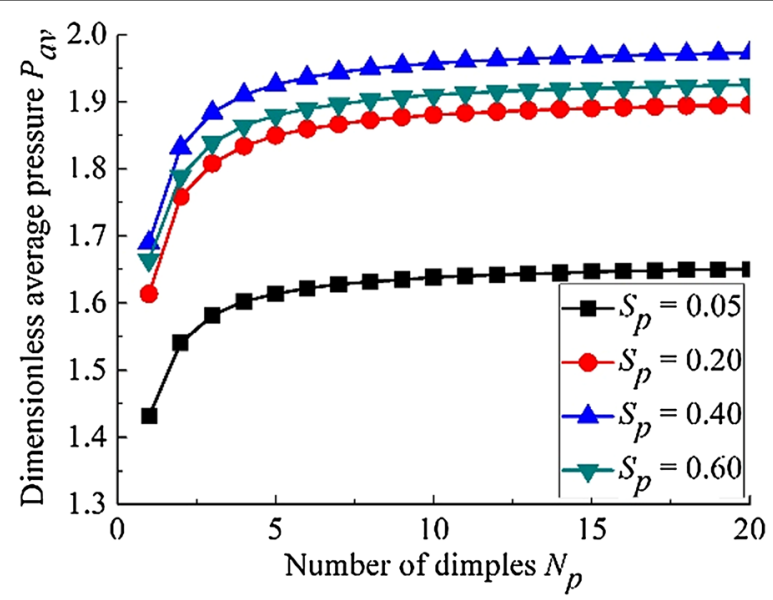

Figure 7 Dimensionless average pressure $P_{a v}$ vs. number of dimples $N_{p}$

\subsection{Effect of Surface Roughness on Dimensionless Average Pressure}

Figure 8 shows the hydrodynamic pressure over dimples column for smooth textured surfaces at $\sigma=0$. From this figure, the film pressure distribution is completely smooth, which is due to the fact that the hydrodynamic pressure is only generated by micro-dimples. By comparing Figure 7 and Figure 8, it is shown that there are ripples on the surface of pressure distribution for the sake of roughness. Besides, the hydrodynamic pressure is reduced by roughness. Therefore, the effect of surface roughness should be considered in the process of numerical analysis the hydrodynamic lubrication of surface textures.

The effect of the roughness ratio, $\sigma$, on the dimensionless average pressure, $P_{a v}$, is presented in Figure 9 for several values of the dimple depth ratio, $\delta$. In the case of $\delta=0$, there exists an optimum dimple depth to maximize the hydrodynamic lubrication of the untextured rough surface. When the dimple depth ratio $\delta>0$, the average pressure decreases with the increase of the roughness ratio, which would be expected since the roughness greatly suppresses the hydrodynamic effect of dimples. Therefore, the roughness ratio should be as small as possible in the engineering practice of the textured surfaces. Based on the above analysis, the effect of roughness cannot be neglected in investigating the hydrodynamic lubrication of surface texture by means of numerical analysis.

In this paper, the wavelengths have been fixed at $w_{x}=w_{y}$ in the following cases, and hence the roughness can be considered nearly isotropic. Further investigation reveals that for a range of dimple depth ratio values the wavelengths of sinusoidal roughness have an effect on the hydrodynamic pressure, as shown in Figure 10. The average pressure varies almost linearly with the wavelengths on the untextured surface. However, the average pressure almost does not change with the wavelengths even as $\delta=0.5$, particularly for the higher value of $\delta$. In these cases, the dimples play a major role in generating hydrodynamic pressure. Therefore, it is appropriate to describe the roughness textured surfaces with dimples.

\subsection{Effect of Micro-dimple Dimple on Dimensionless Average Pressure}

The influence of the dimple depth ratio on the dimensionless average pressure for various roughness ratios is presented in Figure 11. From Figure 11, it is evident that there is an optimum dimple depth ratio to maximize the average pressure for any roughness ratio. However, the roughness ratio rarely affects the optimum dimple depth ratio. 


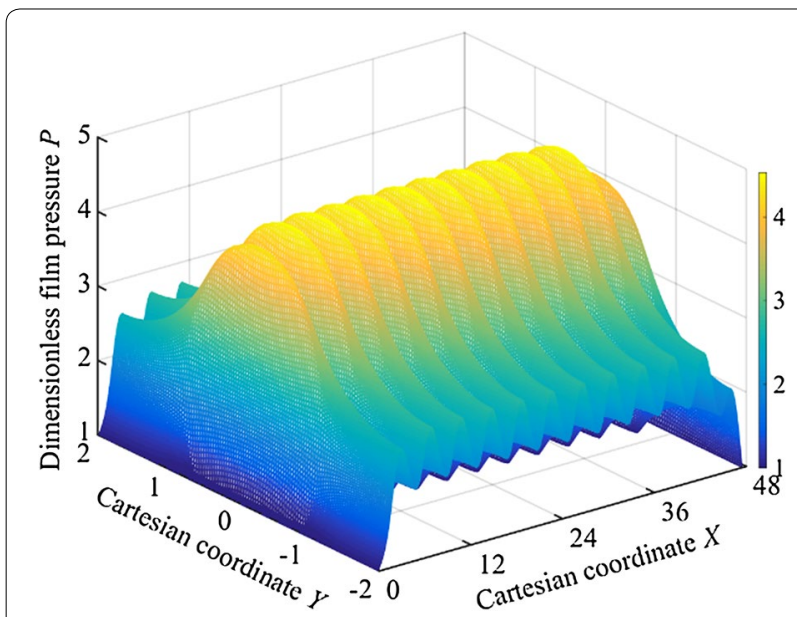

Figure 8 Hydrodynamic pressure over dimples column for $\sigma=0$

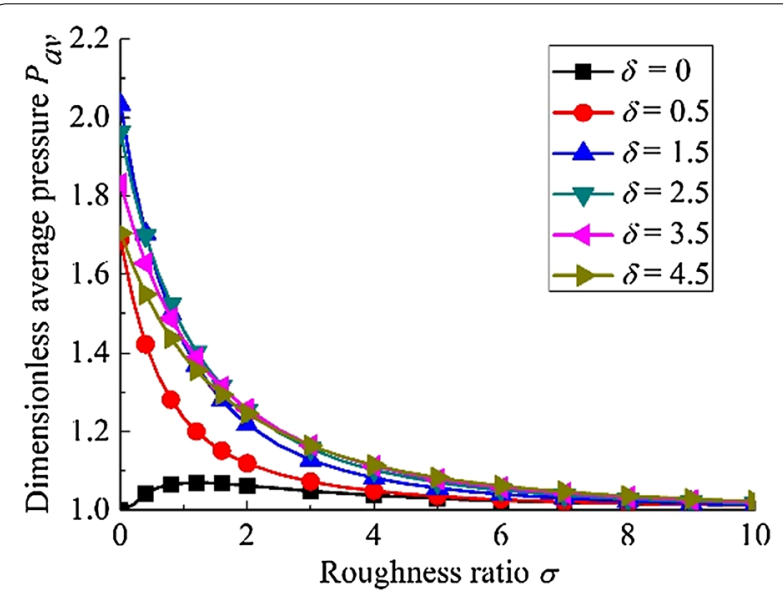

Figure 9 Dimensionless average pressure $P_{a v}$ vs. roughness ratio $\sigma$

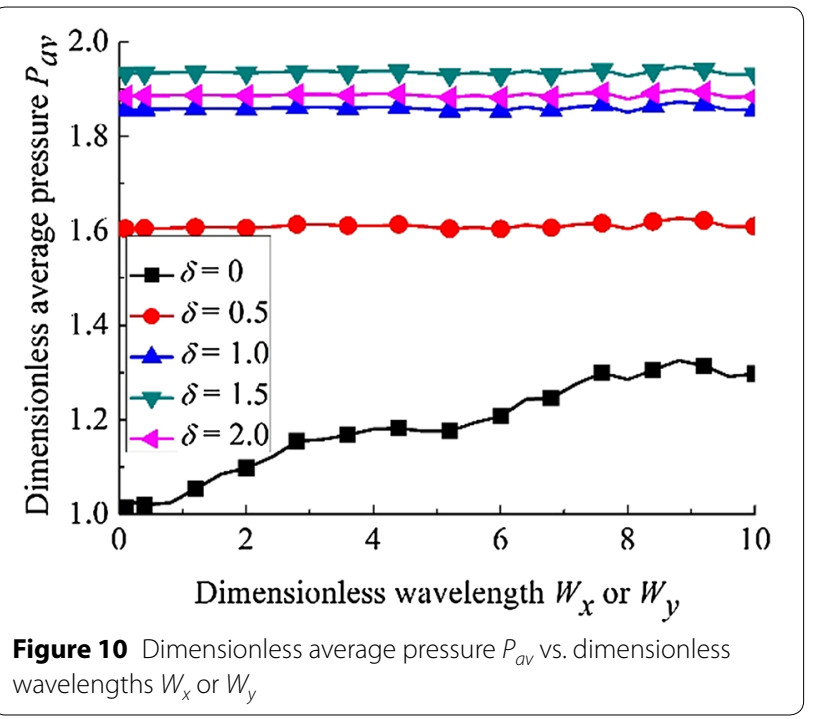

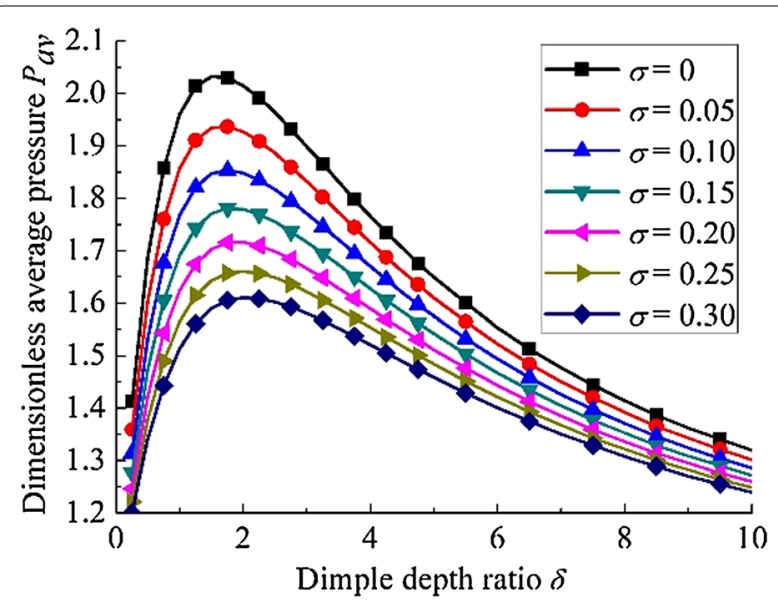

Figure 11 Dimensionless average pressure $P_{a v}$ vs. dimple depth ratio

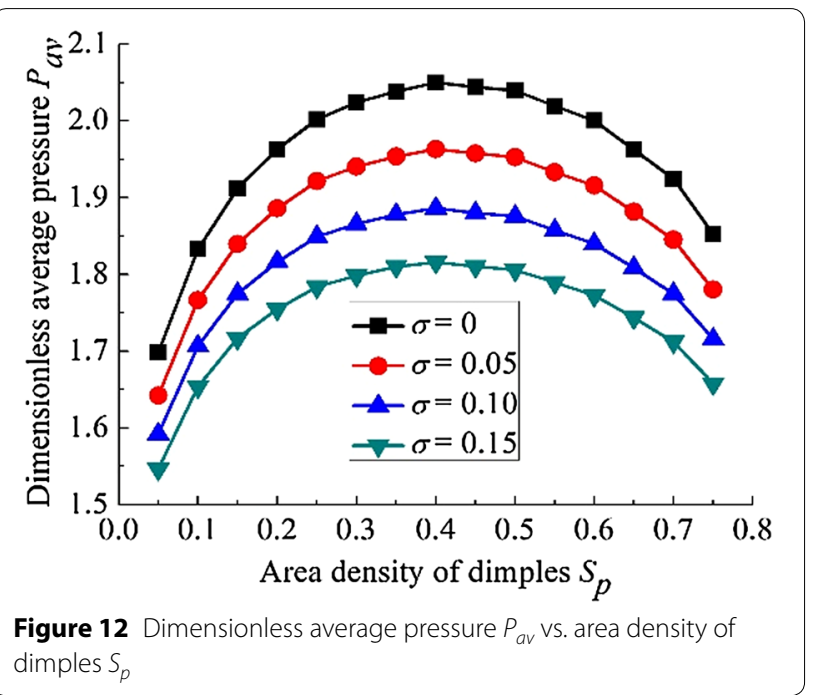

Figure 12 shows the influence of the area density on the dimensionless average pressure for several values of the dimple ratio. It is noticed that the value of $P_{a v}$ gradually increases with the increase of area density, going for a maximum at about $S_{p}=0.4$. Then the average pressure gradually decreases with the increase of area density. Besides, the dimple depth has little impact on the optimum area density of the dimple.

\section{Conclusions}

1. The influence of the interaction between adjacent micro-dimples of the hydrodynamic pressure is of significance, whether along or perpendicular to the direction of the motion. As a result the interactions between neighboring dimples cannot be simply omitted in either direction. 
2. The influence of roughness on the hydrodynamic pressure of textured surface is significant. The hydrodynamic pressure decreases with the increment of the roughness ratio because the roughness greatly suppresses the hydrodynamic effect of dimples.

3. There are the optimum values of the dimple depth ratio and area density to maximize the hydrodynamic pressure at any roughness ratio, respectively. The roughness ratio has no obvious effect on the optimum value of dimple depth ratio and the area density.

\section{Authors' Contributions}

J-HJ carried out the numerical simulation and manuscript writing. C-WG carried out the analysis of the numerical results. Y-HF revised the manuscript. All authors read and approved the final manuscript.

\section{Authors' Information}

Jing-Hu Ji, born in 1982, is currently an associate professor at School of Mechanical Engineering, Jiangsu University, China. He received his PhD degree from Jiangsu University, China, in 2012. His research interests include surface engineering, tribology, laser surface texturing.

Cai-Wei Guan, born in 1991, is currently a master candidate at School of Mechanical Engineering, Jiangsu University, China. Her research interests include tribology, laser surface texturing.

Yong-Hong Fu, born in 1965, is currently a professor at School of Mechanical Engineering, Jiangsu University, China. He received his PhD degree from Jiangsu University, China, in 2008. His research interests include tribology, surface engineering, laser surface texturing, internal combustion engine, mechanical seal and die technology.

\section{Competing Interests}

The authors declare that they have no competing interests.

\section{Funding}

Supported by National Natural Science Foundation of China (Grant Nos. 51305168, 51375211, 51375213), Jiangsu Provincial Natural Science Foundation of China (Grant No. BK20130524), and Research Foundation for Advanced Talents of Jiangsu University, China (Grant No. 13JDG090).

\section{Publisher's Note}

Springer Nature remains neutral with regard to jurisdictional claims in published maps and institutional affiliations.

Received: 20 February 2017 Accepted: 9 August 2018

Published online: 20 August 2018

\section{References}

[1] U Sudeep, N Tandon, R K Pandey. Performance of lubricated rolling/ sliding concentrated contacts with surface textures: A review. Journal of Tribology-Transactions of the ASME, 2015, 137(3): 031501.

[2] D Gropper, L Wang, T J Harvey. Hydrodynamic lubrication of textured surfaces: A review of modeling techniques and key findings. Tribology International, 2016, 94: 509-529.

[3] I Etsion. Improving tribological performance of mechanical components by laser surface texturing. Tribology Letters, 2004, 17(4): 733-737.

[4] C C Ji, H Zhu, W Jiang, et al. Running-in test and fractal methodology for worn surface topography characterization. Chinese Journal of Mechanical Engineering, 2010, 23(5): 600-605.

[5] X L Wang, K Adachi, K Otsuka, et al. Optimization of the surface texture for silicon carbide sliding in water. Applied Surface Science, 2006, 253(3): 1282-1286.
[6] TWoloszynski, P Podsiadlo, GW Stachowiak. Evaluation of discretization and integration methods for the analysis of hydrodynamic bearings with and without surface texturing. Tribology Letters, 2013, 51(1): 25-47.

[7] Q J Wang, D Zhu. Virtual texturing: Modeling the performance of lubricated contacts of engineered surfaces. Journal of Tribology-Transactions of the ASME, 2005, 127(4): 722-728.

[8] NTala-Ighil, P Maspeyrot, M Fillon, et al. Effect of surface texture on journal-bearing characteristics under steady-state operating conditions. Proceedings of the Institution of Mechanical Engineers, Part J: Journal of Engineering Tribology, 2007, 221(J6): 623-633.

[9] A Ronen, I Etsion, Y Kligerman. Friction-reducing surface-texturing in reciprocating automotive components. Tribology Transactions, 2001, 44(3): 359-366.

[10] R B Siripuram, L S Stephens. Effect of deterministic asperity geometry on hydrodynamic lubrication. Journal of Tribology-Transactions of the ASME, 2004, 126(3): 527-534.

[11] HW Yu, X L Wang, F Zhou. Geometric shape effects of surface texture on the generation of hydrodynamic pressure between conformal contacting surfaces. Tribology Letters, 2010, 37(2): 123-130.

[12] J Han, L Fang, J P Sun, et al. Hydrodynamic lubrication of microdimple textured surface using three-dimensional CFD. Tribology Transactions, 2010, 53(6): 860-870.

[13] D Zhu, T Nanbu, N Ren, et al. Model-based virtual surface texturing for concentrated conformal-contact lubrication. Proceedings of the Institution of Mechanical Engineers, Part J: Journal of Engineering Tribology, 2010, 224(J8): 685-696.

[14] V Brizmer, Y Kligerman, I Etsion. A laser surface textured parallel thrust bearing. Tribology Transactions, 2003, 46(3): 397-403.

[15] N Tala-Ighil, M Fillon, TP Maspeyrot. Effect of textured area on the performances of a hydrodynamic journal bearing. Tribology International, 2011, 44(3): 211-219.

[16] M B Dobrica, M Fillon, M D Pascovici, et al. Optimizing surface texture for hydrodynamic lubricated contacts using a mass-conserving numerical approach. Proceedings of the Institution of Mechanical Engineers, Part J: Journal of Engineering Tribology, 2010, 224(J8): 737-750.

[17] H B Liu, Y G Meng. Hydrodynamic lubrication analysis of textured surfaces with the domain decomposition method-effect of textured distribution patterns. Tribology, 2007, 27(6): 555-561. (in Chinese)

[18] T Nanbu, N Ren, Y Yasuda, et al. Micro-textures in concentrated conformal-contact lubrication: Effects of textured bottom shape and surface relative motion. Tribology Letters, 2008, 29(3): 241-252.

[19] C Shen, M M Khonsari. Effect of dimple's internal structure on hydrodynamic lubrication. Tribology Letters, 2013, 52(3): 415-430.

[20] L Wang, W Z Wang, H Wang, et al. Numerical analysis on the factors affecting the hydrodynamic performance for the parallel surfaces with microtextures. Journal of Tribology-Transactions of the ASME, 2014, 136(2): 021702.

[21] H P Yao, P Huang. Load carrying capacity of parallel movement lubricated rough surfaces. Tribology, 2008, 28(2): 150-154. (in Chinese)

[22] D Zhu, Q J Wang. Effect of roughness orientation on elastohydrodynamic lubrication film thickness. Journal of Tribology- Transactions of the ASME, 2013, 135(3): 031501.

[23] Y Qiu, M M Khonsari. Performance analysis of full-film textured surfaces with consideration of roughness effects. Journal of Tribology-Transactions of the ASME, 2011, 133(2): 021704.

[24] Y Z Hu, D Zhu. A full numerical solution to the mixed lubrication in point contacts. Journal of Tribology-Transactions of the ASME, 2000, 122(1): 1-9.

[25] Z R Zhou. The development frontlines of tribology. Beijing: Science Press, 2006. (in Chinese)

[26] H G Elrod. A cavitation algorithm. Journal of Lubrication Technology-Transactions of the ASME, 1981, 103(3): 350-354.

[27] C H Venner, A A Lubrecht. Multigrid techniques: A fast and efficient method for the numerical simulation of elastohydrodynamic lubricated point contact problems. Proceedings of the Institution of Mechanical Engineers, Part J: Journal of Engineering Tribology, 2000, 214(J1): 43-62.

[28] M Fesanghary, M M Khonsari. A modification of the switch function in the Elrod cavitation algorithm. Journal of Tribology-Transactions of the ASME, 2011, 133(2): 024501.

[29] J H Ji, Y H Fu, Q S Bi. The influence of partially textured slider with orientation ellipse dimples on the behavior of hydrodynamic lubrication. Industrial Lubrication and Tribology, 2014, 66(2): 161-167. 